\title{
Shear-Enhanced Transfer Printing of Conducting Polymer Thin Films
}

\author{
Pratik Sen, ${ }^{\dagger}$ Yuan Xiong, ${ }^{\S}$ Qianqian Zhang, ${ }^{\| \odot}$ Sungjune Park, ${ }^{\perp}$ Wei You, ${ }^{\| \odot ~ H a r a l d ~ A d e, ~}{ }^{\S}$ \\ Michael W. Kudenov, ${ }^{\ddagger}$ and Brendan T. O’Connor*,† \\ ${ }^{\dagger}$ Department of Mechanical and Aerospace Engineering, ${ }^{\ddagger}$ Department of Electrical and Computer Engineering, ${ }^{\S}$ Department of \\ Physics, Organic and Carbon Electronics Lab (ORaCEL), and ${ }^{\perp}$ Chemical and Biomolecular Engineering, North Carolina State \\ University, Raleigh, North Carolina 27695, United States \\ "Department of Chemistry, University of North Carolina at Chapel Hill, Chapel Hill, North Carolina 27599, United States
}

ABSTRACT: Polymer conductors that are solution-processable provide an opportunity to realize low-cost organic electronics. However, coating sequential layers can be hindered by poor surface wetting or dissolution of underlying

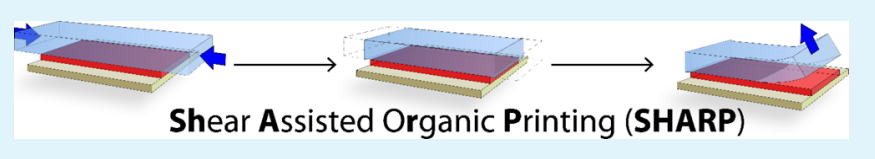

layers. This has led to the use of transfer printing where solid film inks are transferred from a donor substrate to partially fabricated devices using a stamp. This approach typically requires favorable adhesion differences between the stamp, ink, and receiving substrate. Here, we present a shear-assisted organic printing (SHARP) technique that employs a shear load on a postless polydimethylsiloxane (PDMS) elastomer stamp to print large-area polymer films that can overcome large unfavorable adhesion differences between the stamp and receiving substrate. We explore the limits of this process by transfer printing poly(3,4-ethylenedioxythiophene):polystyrene sulfonate (PEDOT:PSS) films with varied formulation that tune the adhesive fracture energy. Using this platform, we show that the SHARP process is able to overcome a 10-fold unfavorable adhesion differential without the use of a patterned PDMS stamp, enabling large-area printing. The SHARP approach is then used to print PEDOT:PSS films in the fabrication of high-performance semitransparent organic solar cells.

KEYWORDS: transfer printing, adhesion, PEDOT:PSS, surfactant, organic photovoltaics

\section{INTRODUCTION}

Conjugated polymers are attractive materials for large-area electronics because of their unique optoelectronic properties and potential to reduce device costs. They are typically solution-cast and part of a number of heterogeneous layers that make up a device. However, solution-casting sequential layers can be challenging because of dissolution of underlying layers or because of poor wettability of the ink on the surface of interest. This has led to transfer printing methods where solid film "inks" are transferred from a donor substrate to a receiving substrate using an elastomer stamp. ${ }^{1-6}$ The success of this approach has led to its broad applications in processing organic electronics. ${ }^{4,7-10}$ The transfer printing technique typically relies on increasing the adhesion of the ink from the donor substrate, to the stamp, and finally to the receiving substrate. ${ }^{7,11}$ When this criterion is not met, modifications to the transfer printing process are often employed including the use of sacrificial layers, ${ }^{12-14}$ the use of heat, ${ }^{15-17}$ or modifying the surface of the film or stamp through $\mathrm{UV} /$ ozone or $\mathrm{O}_{2}$ plasma treatment. ${ }^{7,18-20}$ However, these methods can be invasive and negatively impact the final device. Alternatively, various mechanical loading protocols can be employed to improve transfer printing success. ${ }^{2,5,11,21,22}$ In particular, Carlson et al. described a transfer printing technique that applies a shear load to a polydimethylsiloxane (PDMS) elastomer stamp with posts to enable printing of $\mathrm{Si}$ nanomembranes. $^{21,23}$ The shear load on the PDMS reduces the normal force required for delamination and creates a moment that concentrates a tensile stress at the leading edge of the stamp post. ${ }^{22,23}$ This approach was shown to overcome unfavorable adhesion differences between the stamp/ink and ink/receiving substrate. However, this method was only demonstrated for stiff silicon membranes that were $\sim 0.01$ $\mathrm{mm}^{2}$ in area and over $300 \mathrm{~nm}$ thick. ${ }^{21}$ As they reduced the film thickness, the transfer printing process was unsuccessful. This poses a challenge for applying this approach to thin-film organic electronic films that are comparatively soft and thin and where large areas are often desired. In order to address these challenges, we developed a modified shear-based printing technique that uses an unpatterned prestrained PDMS stamp to print large-area polymer thin films. We refer to this approach as shear-assisted organic printing (SHARP). We have previously used SHARP to produce devices difficult to realize with solution coating ${ }^{24-28}$ and to probe structure-property relationships in organic semiconductors. ${ }^{29-31}$ For example, this approach was used to transfer print polymer semiconductor films in organic transistors showing similar device characteristics to direct solution-cast films. ${ }^{24,25}$ It was shown that films could be repeatedly retrieved and printed from a low surface energy substrate multiple times without impacting charge transport. $^{25}$ This approach has also been used to consecutively print multiple polymer and small-molecule films with sharp

Received: June 15, 2018

Accepted: August 27, 2018

Published: August 27, 2018 


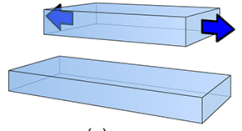

(a)

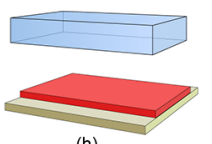

(h)

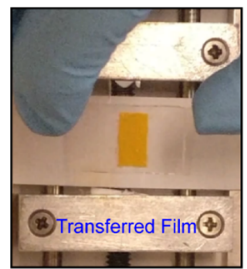

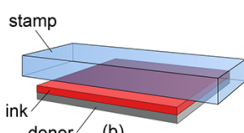

donor (b)

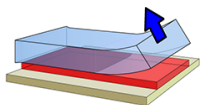

(g)

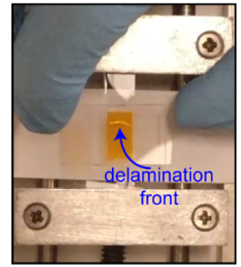

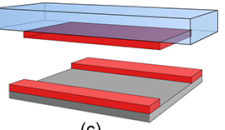

(c)
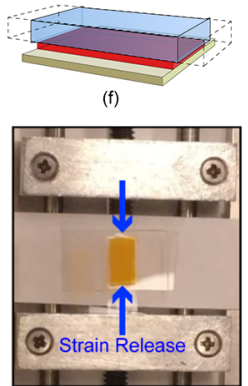
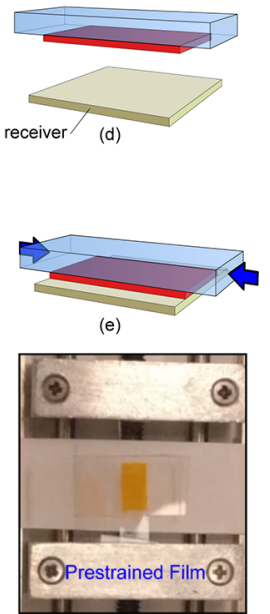

Figure 1. Illustration of the SHARP technique with the following steps: (a) strain applied to the elastomer stamp, (b) lamination of the stamp onto the ink, (c) transfer of the ink to the stamp assisted by the quick removal of the donor substrate, (d,e) stamp moved and laminated to the receiving substrate, (f) removal of the strain applied to the stamp, (g) normal force applied to the leading edge of the stamp, resulting in delamination of the ink, and (h) removal of the stamp, leaving the ink on the receiving substrate. Pictures of the process are also provided that correspond to illustrations $(\mathrm{e}-\mathrm{h})$.

interfaces, ${ }^{29,30}$ including the ability to print sharp planar heterojunction organic photovoltaic (OPV) cells. ${ }^{27}$ Although the SHARP technique has been used to fabricate a number of devices and structures, the capabilities of this approach have not been considered in detail. In this paper, we explore the adhesion limits of the SHARP technique and use this approach to fabricate OPV cells. We focus on printing the widely used conducting polymer poly(3,4-ethylenedioxythiophene):polystyrene sulfonate (PEDOT:PSS) and tune the adhesion of the films by varying the PEDOT:PSS formulation. We show that the SHARP method overcomes an unfavorable work of adhesion ratio between the stamp/ink and ink/receiver of approximately 10 without the use of heat or stamp modification. We then apply the SHARP method to print PEDOT:PSS films as transparent electrodes to realize highperformance semitransparent OPV cells.

PEDOT:PSS is chosen as a model ink for its wide use and more practically to meet a need for effective processing organic electronics. For example, in semitransparent OPV cells, there is a need for a transparent electrode capping the device. ${ }^{32-37}$ Conducting polymers are particularly attractive for this top electrode because of their low-cost, low-temperature processing, and mechanical flexibility. The most common polymer investigated has been PEDOT:PSS because of its high transparency, high conductivity, and stability. However, it is a challenge to solution-cast PEDOT:PSS as a top electrode in organic electronic devices because of poor surface wetting ${ }^{38,39}$ and incompatibility of processing methods used to improve the film conductivity. ${ }^{40}$ Specifically, to achieve high conductivity, PEDOT:PSS can be treated with organic solvents such as dimethyl sulfoxide (DMSO) and ethylene glycol, which increases the conductivities of the film by 2 orders of magnitude. ${ }^{41-48}$ However, the additive may diffuse into underlying layers during solution processing. The increasing conductivity may also be achieved by post-treating the films with acids. ${ }^{44}$ However, acid treatments cannot be performed on PEDOT:PSS with underlying organic semiconductor layers. Because of these challenges, there have been several demonstrations of PEDOT:PSS films that are cast and optimized on a donor substrate and then transfer-printed during device fabrication. ${ }^{40,48-50}$ To successfully transfer print the PEDOT:PSS films, external plasma or UV/ozone treatment has been used to increase the interfacial surface energy between the film and receiving substrate. ${ }^{9,20,51,52}$ Another approach has been to apply heat during the printing process once the film is in contact with the receiving substrate. ${ }^{32,53-55}$ The SHARP approach mitigates these additional processing steps that potentially damage the underlying layers. While PEDOT:PSS is used as the ink in this study, the SHARP process is widely applicable to other polymers and is a powerful tool to produce a range of devices.

\section{EXPERIMENTAL DETAILS}

2.1. SHARP Method. The SHARP technique is illustrated in Figure 1. The process starts by placing the stamp in a custom strain stage and straining the stamp by at least $10 \%$. The PDMS stamp is then laminated onto the ink that is on a donor substrate. The donor substrate is then removed rapidly from the stamp to promote transfer of the ink from the donor to the stamp by exploiting the ratedependent adhesive properties of the stamp. ${ }^{56}$ The stamp/ink composite is then laminated onto the receiving substrate. The strain in the stamp is then reduced by approximately $5-10 \%$. This results in a shear load across the stamp/ink interface and a moment at the leading edge of the adhered stamp. After reducing the strain on the stamp, a small normal tensile force is applied at one side of the stamp that results in delamination of the ink from the stamp at the leading edge of the ink. Once the stamp delaminates from the edge of the ink, a delamination front propagates across the ink and it is effectively transferred to the receiving substrate. This process significantly reduces the normal force required to reach the adhesive fracture energy at the stamp/ink interface, enabling film transfer from the stamp to the receiving substrate. A video of this process is provided in the Supporting Information, with snapshots of the process given in Figure 1.

2.2. PEDOT:PSS Film Preparation and Characterization. PEDOT:PSS was chosen as the ink to study the limits of the SHARP technique because of its tunable adhesive properties through the use of surfactants and adhesion promoters. ${ }^{39,43,57,58}$ We focused on the high-conductivity Clevios PH1000 PEDOT:PSS and modified the adhesive properties using two primary approaches: (1) the addition of DuPont Capstone FS30 fluorosurfactant ${ }^{43,59}$ and (2) blending with Clevios CPP105D PEDOT:PSS. CPP105D contains Dynol 604, an acetylenic glycol-based nonionic surfactant, and Silquest A187, an 
Table 1. PEDOT:PSS Formulations Considered with Film Properties

\begin{tabular}{|c|c|c|c|c|c|}
\hline name & formulation $^{a}$ & thickness (nm) & conductivity spun-cast $(\mathrm{S} / \mathrm{cm})$ & conductivity printed $^{b}(\mathrm{~S} / \mathrm{cm})$ & contact angle (deg) \\
\hline PH1000 & PH1000 & 104 & $795 \pm 71$ & & $110.4 \pm 2$ \\
\hline FS30-1\% & $\mathrm{PH} 1000+1 \% \mathrm{FS} 30$ & 131 & $604 \pm 3$ & $574 \pm 19$ & $45.1 \pm 5$ \\
\hline FS30-5\% & PH1000 + 5\% FS30 & 160 & $436 \pm 2$ & $443 \pm 14$ & $23.1 \pm 4$ \\
\hline FS30-10\% & PH1000 + 10\% FS30 & 195 & $358 \pm 28$ & $369 \pm 5$ & $20.0 \pm 5$ \\
\hline CPP-1:0 & CPP105D & 187 & $43 \pm 3$ & NA & $33.3 \pm 5$ \\
\hline СРР-3:1 & CPP105D:PH1000 (3:1) & 191 & $202 \pm 3$ & NA & $33.2 \pm 8$ \\
\hline CPP-1:1 & CPP105D:PH1000 (1:1) & 206 & $341 \pm 13$ & NA & $33.5 \pm 3$ \\
\hline CРP-1:3 & CPP105D:PH1000 (1:3) & 205 & $436 \pm 24$ & $415 \pm 2$ & $33.7 \pm 2$ \\
\hline MSA & PH1000 + MSA & 74 & $843 \pm 32$ & $849 \pm 70$ & NA \\
\hline
\end{tabular}

${ }^{a}$ The added FS30 is by weight percent, and the ratio of CPP105D:PH1000 is by volume. ${ }^{b}$ Printed conductivity was measured for films printed from OTS-Si to glass substrates.

epoxy-functionalized silane, which has preferential adhesion properties with silicone materials such as PDMS. ${ }^{60}$ Capstone FS30 was added to the PH1000 PEDOT:PSS solution at 1, 5, or $10 \mathrm{wt} \%$. CPP 105D PEDOT:PSS was mixed with PH1000 PEDOT:PSS at volume ratios of 3:1, 1:1, and 1:3. DMSO was also added at $5 \mathrm{wt} \%$ to these solutions to increase the conductivity. ${ }^{61}$ The solutions were spun-cast onto octyltrichlorosilane (OTS)-treated $\mathrm{Si}$ at $1000 \mathrm{rpm}$ for $30 \mathrm{~s}$. The cast films were then annealed at $150{ }^{\circ} \mathrm{C}$ for $30 \mathrm{~min}$, followed by vacuum drying the films for $10 \mathrm{~h}$ at $1 \times 10^{-6}$ mbars. The OTS-treated $\mathrm{Si}$ was used to provide a low adhesion donor substrate. Finally, PH1000 PEDOT:PSS films treated with methane sulfonic acid (MSA) were also considered. The MSA process is known to significantly increase the electrical conductivity of the film and results in the ability to retrieve the film from a glass donor substrate onto a PDMS stamp. ${ }^{40,50}$ In this case, PH1000 PEDOT:PSS with 5 wt \% DMSO was spun-cast on glass substrates at $1500 \mathrm{rpm}$ for $30 \mathrm{~s}$. The films were then dried at $120{ }^{\circ} \mathrm{C}$ for $20 \mathrm{~min}$. This was followed by covering the substrate with $100 \mu \mathrm{L}$ of MSA and thermally annealing at $160{ }^{\circ} \mathrm{C}$ for $4 \mathrm{~min}$. The acid was removed by immersing the substrate in deionized water and in isopropyl alcohol, before being dried at 160 ${ }^{\circ} \mathrm{C}$ for $1 \mathrm{~min}$. There are a total of nine different PEDOT:PSS-based films considered, with processing conditions and film thickness summarized in Table 1. Also included in Table 1 are shorthand names for the formulations that will be used throughout the rest of the paper. The film thicknesses were determined by spectroscopic ellipsometry. The film transmittance was measured using an Ocean Optics Jazz spectrometer.

2.3. Adhesive Fracture Energy. Generally, the ability to transfer print a polymer film is dependent on the relative adhesive fracture energy between the donor and ink $\left(G_{\mathrm{c}}^{\text {donor/ink }}\right)$, the stamp and ink $\left(G_{\mathrm{c}}^{\text {stamp/ink }}\right)$, and the ink and receiver $\left(G_{\mathrm{c}}^{\text {ink/receiver }}\right)^{11}$

$$
G_{\mathrm{c}}^{\text {donor } / \text { ink }}<G_{\mathrm{c}}^{\text {stamp } / \text { ink }}, \text { for printing from donor onto stamp }
$$

$$
G_{\mathrm{c}}^{\text {ink/receiver }}>G_{\mathrm{c}}^{\text {stamp/ink }} \text {, for printing from stamp onto receiver }
$$

In this study, the donor substrate is either OTS-Si or untreated glass, and the receiving substrate is OTS-Si. OTS-Si was chosen for the receiving substrate because of its low surface energy, making it a challenging surface to print the PEDOT:PSS ink. The adhesion fracture energy between the stamp/ink and ink/receiver was measured using peel tests. For fracture energy from the PDMS stamp, the stamp is adhered to a glass substrate and delamination was conducted slowly to minimize viscoelastic effects. The PDMS (Sylgard 184) stamp was prepared in a $15: 1$ ratio of base to crosslinker and cured for $12 \mathrm{~h}$ under vacuum at $60{ }^{\circ} \mathrm{C}$. For all peel tests, a backing tape ( $3 \mathrm{M}$ double-sided tape) was laminated on the PEDOT:PSS surface and attached to the grips of an Instron 5943 tensile tester. The average applied load during delamination was then used to obtain the adhesive fracture energy by ${ }^{62}$

$$
G=\frac{(P / b)^{2}}{2 E h}+\frac{P}{b}(1-\cos \theta)
$$

where $P$ is the peel force, $b$ is the width of the peeling arm, $E$ and $h$ are the composite elastic modulus and height of the backing layer and film, and $\theta$ is the peel angle. Assuming high tensile stiffness and low bending stiffness of the bending arm, the first term in eq 3 is neglected. The peel angle $\theta$ was approximately $90^{\circ}$. The contact angle of the inks on OTS-treated $\mathrm{Si}$ substrates was also measured to consider surface wetting and its relationship to adhesion of the solid films to the stamp. Contact angles were measured using a rame-hart standard goniometer (model 200-U1).

2.4. Device Fabrication. Two OPV active layers are considered with the printed PEDOT:PSS electrode to achieve semitransparent devices. Control devices with a reflective top metal electrode are also considered.

2.4.1. P3HT:PC ${ }_{61} B M$ Device Preparation. A thin layer of polyethylenimine (PEIE), $80 \%$ ethoxylated solution, was spun-cast onto patterned indium tin oxide (ITO)-coated glass substrates at $5000 \mathrm{rpm}$ for $60 \mathrm{~s}$ in air, followed by drying the film at $100{ }^{\circ} \mathrm{C}$ for 10 $\mathrm{min}$. This layer reduces the work function of ITO, allowing for an inverted OPV architecture. ${ }^{63}$ Regioregular poly(3-hexylthiophene) (P3HT, Rieke Metals) and phenyl-C61-butyric acid methyl ester (PCBM, Nano-C) with a 60:40 mass ratio were dissolved in dichlorobenzene at a total concentration of $34 \mathrm{mg} / \mathrm{mL}$. The films were spun-cast at $700 \mathrm{rpm}$ for $60 \mathrm{~s}$ in a nitrogen-filled glovebox. Control device films were then annealed at $130^{\circ} \mathrm{C}$ for $10 \mathrm{~min}$.

2.4.2. FTAZ:IT-M Device Preparation. A ZnO film was cast onto an ITO substrate at $5000 \mathrm{rpm}$ for $60 \mathrm{~s}$ using a previously described method to form an inverted architecture. ${ }^{64} \operatorname{Poly}(4,8$-bis $(3-$ butylnonyl)benzo[1,2-b:4,5-b] dithiophen-2-yl)thiophen-2-yl-2-(2-butyloctyl)-5,6-difluoro-7-(thiophen-2-yl)-2H-benzo[d][1,2,3] triazole (FTAZ) was synthesized using previously reported methods, ${ }^{65}$ and (3,9-bis(2-methylene-((3-(1,1-dicyanomethylene)-6/7-methyl)-indanone))-5,5,11,11-tetrakis (4-hexylphenyl)-dithieno[2,3-d:2',3'-d']-sindaceno $\left[1,2-b: 5,6-b^{\prime}\right]$ dithiophene) (IT-M) was purchased from 1 Material. FTAZ and IT-M were dissolved in toluene with a mass ratio of $1: 1$ with a total concentration of $10 \mathrm{mg} / \mathrm{mL}$. The solution was cast on the $\mathrm{ZnO}$-coated substrate at $1000 \mathrm{rpm}$ for $60 \mathrm{~s}$. The control film devices were annealed at $150{ }^{\circ} \mathrm{C}$ for $10 \mathrm{~min}$.

2.4.3. Post-Film Deposition. For opaque devices, a top electrode of $12 \mathrm{~nm} \mathrm{MoO}_{3}$, followed by an opaque metal layer of silver or aluminum, was deposited by vacuum thermal evaporation at $10^{-6}$ mbar. For the semitransparent devices, a $10 \mathrm{~nm} \mathrm{MoO}_{3}$ layer was deposited, followed by transfer printing the PEDOT:PSS electrode. A comparison is also made to a solution-cast top PEDOT:PSS electrode. In these cases, the entire stack was annealed at $140{ }^{\circ} \mathrm{C}$ for $30 \mathrm{~min}$. The electrical characteristics of the OPV devices were tested using a Newport $150 \mathrm{~W}$ solar simulator with an AM1.5G filter under 1 sun $\left(100 \mathrm{~mW} / \mathrm{cm}^{2}\right)$ illumination. The active area for the semitransparent devices was approximately $0.025 \mathrm{~cm}^{2}$. 


\section{RESULTS AND DISCUSSION}

3.1. Adhesion Limits of Printed Films. Static contact angle measurements of the various PEDOT:PSS inks on the low surface energy OTS-treated silicon are given in Figure 2.

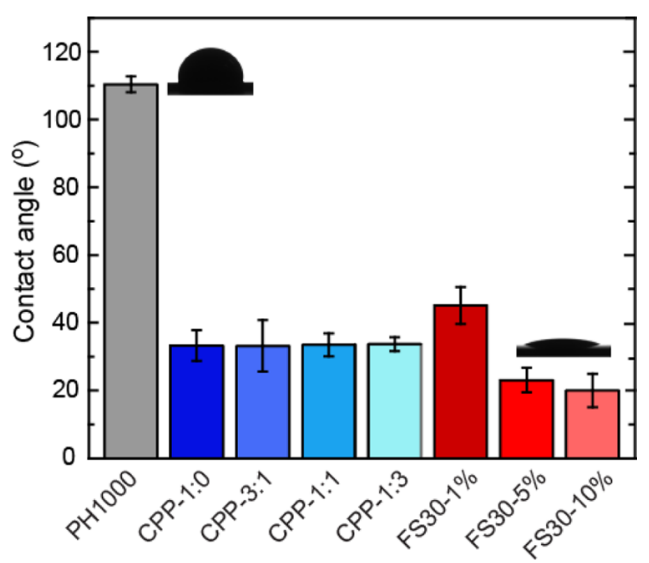

Figure 2. Contact angle measurements for the various PEDOT:PSS formulations listed in Table 1. Inset images are photographs of the PH1000 and FS30-10\% solutions on OTS silicon substrates.

The PH1000 with 5\% DMSO solution was measured as a reference, and its contact angle was found to be $110^{\circ}$. Adding the FS30 surfactant to the PH1000 formulation resulted in a large decrease in contact angle, with a lower angle with increasing FS30 concentration. Adding the CPP105D formulation also resulted in a significant drop in contact angle, which was stable at $\sim 33^{\circ}$ independent of the CPP105D:PH1000 ratios considered. These results are consistent with reducing the surface tension of the ink with the addition of the surfactant. The reduced surface tension improves wetting but should also be reflected in greater adhesion of the film to the substrate. Given the hydrophobic nature of OTS-treated silicon, the donor substrate remains a low adhesion surface. However, the surfactant acts as a plasticizer and will also increase the cohesion of the PEDOT:PSS and adhesion to the PDMS stamp. ${ }^{43,66}$

The nominal adhesive fracture energy between the ink and stamp and the ink and receiver is given in Figure 3. The stamp/ink fracture energies were found to range from 0.75 to $9.0 \mathrm{~J} / \mathrm{m}^{2}$, whereas the average ink/receiver fracture energies were all below $0.80 \mathrm{~J} / \mathrm{m}^{2}$. We found that while the addition of the FS30 surfactant or CPP105D to the PH1000 decreased the ink contact angle relative to neat PH1000, the impact on the adhesive fracture energy was significantly different. The CPP105D-based formulations increased the adhesion to the PDMS stamp significantly more than the FS30-based formulations, which is attributed to the Silquest A187 adhesion promoter in the CPP105D. ${ }^{60}$ In addition, a drop-cast PH1000 film onto the PDMS stamp and OTS-Si substrate had the lowest adhesive fracture energy. MSA-treated PH1000 was found to only have a small effect on the adhesion behavior of PEDOT:PSS. In all cases, the adhesive fracture energy between the stamp and ink was greater than the adhesive fracture energy between the ink and receiver, as shown in Figure 3a. Thus, the nominal cascading fracture energy requirement for simple transfer printing, per eq 2, is not met for any film. This is indeed found to be the case, where transferring the ink from the stamp to the receiver was unsuccessful under normal loading during stamp retrieval for all films considered.
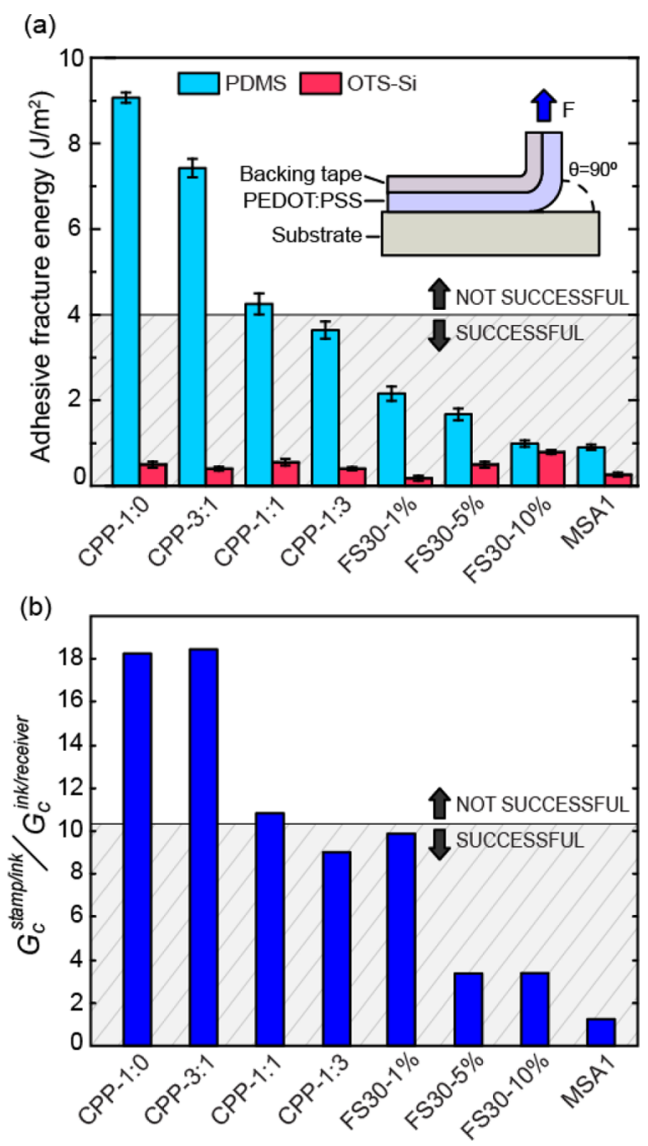

Figure 3. (a) Adhesive fracture energy of various formulations of PEDOT:PSS on PDMS and OTS-treated silicon substrates. The inset is an illustration of the $90^{\circ}$ peel test used to determine the fracture energy. (b) Ratio of the adhesive fracture energy between the stamp/ ink and ink/receiver.

The SHARP technique makes a drastic difference in the ability to print the ink to the receiving substrate. With this approach, the MSA-treated PEDOT:PSS, FS30-5\%, and FS30$10 \%$ all transferred to the receiving surface with near $100 \%$ success rates. Lowering the concentration of FS30 to 1\% decreased the success rate of transfer printing to approximately $70 \%$ based on transferring a minimum of 10 films. When considering the CPP105D formulations, the success rate of the CPP-1:3 blend was approximate $60 \%$. In the failed attempts, the film would often partially transfer, but a portion would not delaminate from the stamp, which would then peel the transferred portion of the film back off the receiving substrate. Further refined control of the printing process should improve the success rate when nearing the printing limits of the process. Increasing the concentration of the CPP105D made transferring films more difficult and the CPP-1:1 and CPP-3:1 blend films did not transfer print successfully with over 10 attempts each. Comparison on the transfer print success to the fracture energy differential $G_{\mathrm{c}}^{\mathrm{stamp}} /$ ink $/ G_{\mathrm{c}}^{\text {ink} / \text { receiver }}$, given in Figure $3 \mathrm{~b}$, shows that the SHARP technique can overcome a 10 -fold negative adhesion ratio. This demonstrates that the SHARP technique is able to overcome large unfavorable fracture energy differences and can be used to print onto very low surface energy substrates. In addition to the ability to print the films, the film morphology and electrical properties should not be negatively impacted. We found that the electrical conductivity of the films, measured using the Van der Pauw technique, are 
similar prior to and after printing, with the results given in Table 1 . The topography of the films after transfer printing is also unchanged, with atomic force microscopy (AFM) images of the FS30-1\% prior to and after transfer printing shown in Figure S1. These results are consistent with previous demonstrations of the films that are transfer-printed onto low adhesion energy surfaces. $25,27,29$

3.2. Thickness Dependence. The previous transfer printing process using shear demonstrated by Carlson et al. had shown a negative relationship between printing success and decrease in the silicon membrane ink thickness. ${ }^{21,22}$ Finite element modeling showed that increasing the thickness of the silicon membrane increases the strain energy release rate at the stamp/ink interface relative to the ink/receiver interface and therefore increases the printing yield. Here, similar experiments were performed to observe whether a similar trend exists for the SHARP technique for printing compliant polymer films. From the results shown in Figure 3, CPP-1:1 and CPP-1:3 films were found to be closest to the transfer printing limit for printing success using the SHARP technique. The CPP-1:1 film was unsuccessful at the film thickness of $206 \mathrm{~nm}$, whereas the CPP-1:3 film was successful at the thickness of $191 \mathrm{~nm}$. These PEDOT:PSS formulations were thus used to consider the transfer print success as a function of film thickness, with the results given in Figure 4. An increase in thickness for CPP-

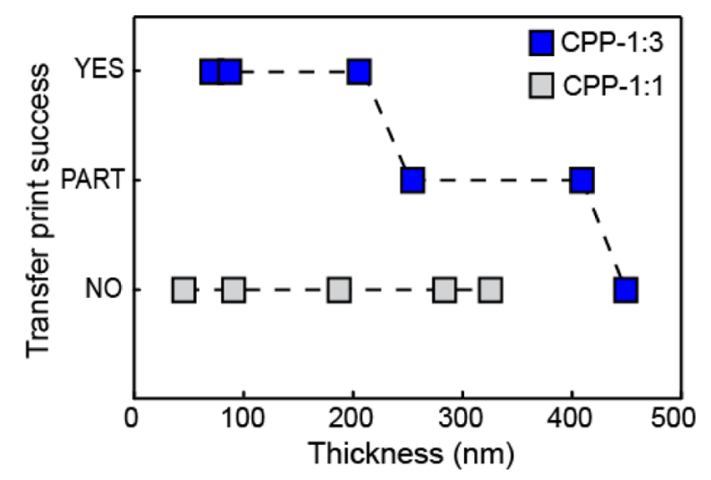

Figure 4. Transfer printing success as a function of film thickness for CPP-1:3 and CPP-1:1 PEDOT:PSS formulation.

1:1 did not show any improvement with printing success with all films failing to print over the thickness range from 50 to 330 $\mathrm{nm}$. The CPP-1:3 films showed a decrease in success rate with increasing thickness. Films with thickness below $250 \mathrm{~nm}$ printed with complete success, films between 250 and $410 \mathrm{~nm}$ printed with partial success including incomplete film transfer in some cases, and films that were $450 \mathrm{~nm}$ thick were not successfully printed. This trend is the opposite of what was found by Carlson et al. for printing $\mathrm{Si}$ membranes. The difference in thickness dependence is currently not fully understood but may be due to a number of competing mechanisms that include differences in the crack initiation between the stamp and ink during the printing processes, differences in film roughness, and possible difference in nominal adhesion between the ink and stamp. In printing the silicon membranes, the stamp posts employed create a similar interface independent of ink thickness. In the SHARP process, the stamp extends beyond the ink and also laminates directly onto the receiving surface. During the shear delamination process, as the stamp delamination front moves across the surface and reaches the edge of the ink, the forces and dynamics will depend on the film thickness. A thicker film may result in stamp dynamics, which promote crack initiation at the ink-receiver interface. Kim-Lee et al. showed that printing success is sensitive to the initial crack length at the ink interfaces. ${ }^{22}$ Small increases in the initial crack length at the ink-receiver interface may result in reduced printing success. Furthermore, the PEDOT:PSS film roughness increases slightly with thickness. This roughness may promote adhesion to the PDMS stamp, lowering the printing success rate. Finally, vertical segregation of the adhesion promoter in the CPP formulation may occur that could result in greater adhesion with increasing film thickness that cannot be ruled out at this time.

3.3. Device Characterization. The utility of the SHARP technique is demonstrated by using it to fabricate semitransparent OPV cells by transfer printing the PEDOT:PSS as a top electrode. As shown in Table 1, the electrical conductivity of the PEDOT:PSS films decreases with added FS30. Thus, we consider devices with the FS30-1\% formulation, which allows for simple spin-casting onto the donor OTS-Si substrate and has a minimal negative impact on electrical conductivity. Two different organic solar cell active layers are considered P3HT:PCBM and FTAZ:IT-M. The FTAZ and IT-M molecular structures are shown in Figure 5a along with the OPV device architecture. The current-voltage characteristics of the semitransparent OPV cells under 1 sun illumination are given in Figure $5 \mathrm{~b}$. The semitransparent OPV cells are compared to devices with reflective metal electrodes. The best power conversion efficiencies (PCEs) for the semitransparent P3HT:PCBM and FTAZ:IT-M OPV cells were 2.37 and $6.02 \%$, respectively. The best PCEs for the control opaque devices were 3.38 and $11.34 \%$, with additional device metrics provided in Table 2. The semitransparent devices had lower short circuit current $\left(J_{\mathrm{SC}}\right)$ and fill factor $(\mathrm{FF})$ compared to the control devices attributed to the transparency and lower conductivity of the PEDOT:PSS electrode relative to the metal electrodes. The FF of the OPV cells with a PEDOT:PSS top electrode is similar to previous demonstrations of similar P3HT:PCBM OPV cells. ${ }^{39,57}$ This includes the PEDOT:PSS layer that is solution-cast and transfer-printed onto the OPV cell. The transfer-printed OPV cell is also compared to a FTAZ:IT-M-based semitransparent OPV cell with spun-cast FS30-1\% top electrodes. The PCE of the spuncast PEDOT:PSS electrode was limited to $1.4 \%$ because of poor FF and $J_{S C}$ (see Figure S2). The poor performance of the solution-cast PEDOT:PSS electrode onto the device may be associated with interactions of the surfactant or DMSO with underlying layers. This highlights the benefit of being able to optimize the film on a donor substrate, followed by device fabrication by transfer printing.

The transmittance of the active layers and semitransparent electrodes is given in Figure 5c. The transmittance of the FS30$1 \%$ PEDOT:PSS film was found to be over $85 \%$ across the visible spectrum. This is comparable to the ITO film used as the counter electrode. The transmittance of the complete device stack is also provided in Figure 5c. As mentioned above, the use of an unpatterned PDMS stamp in the SHARP approach enables the printing of large-area films. As an example, the inset of Figure $5 \mathrm{c}$ shows a large area $(3 \mathrm{~cm} \times 2.5$ $\mathrm{cm}$ ) PEDOT:PSS FS30-1\% film that was printed onto glass, where the film area is only limited by the size of the mechanical stage used in the printing process. 
(a)
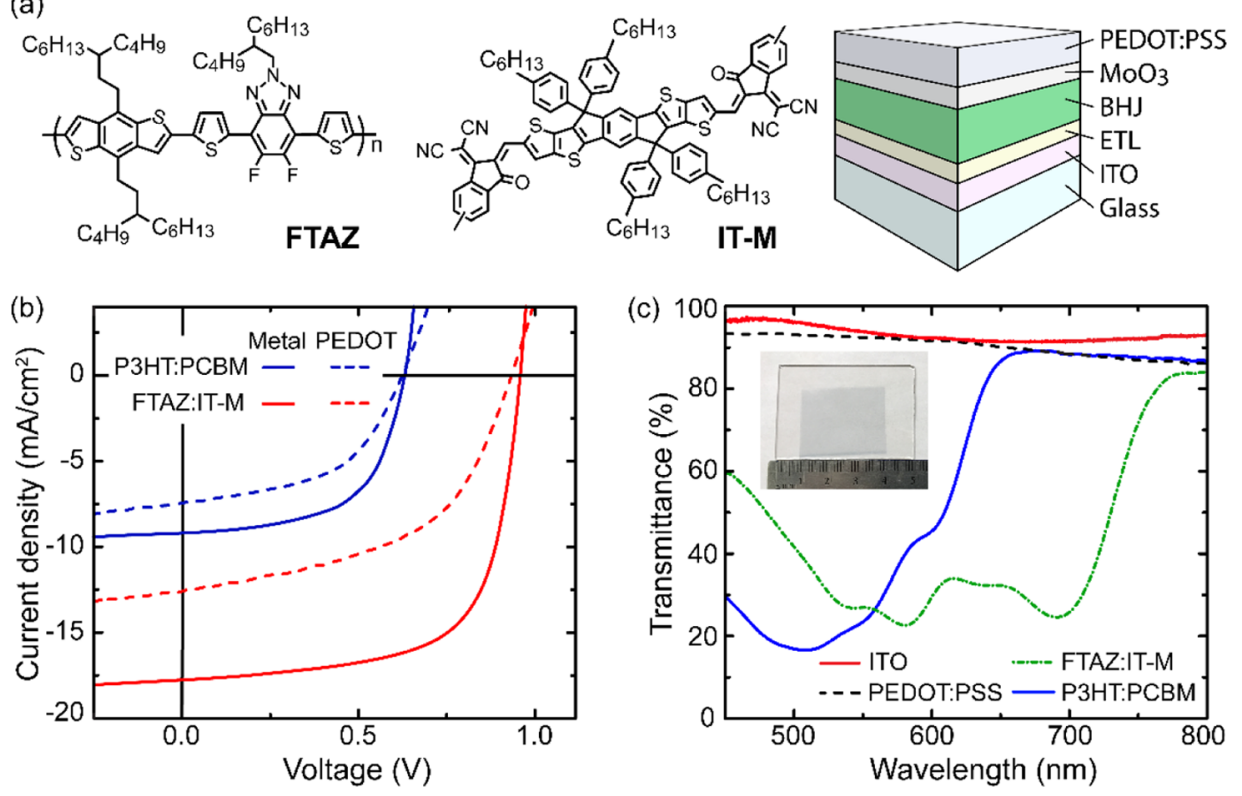

Figure 5. (a) Chemical structure of the polymers FTAZ and IT-M and an illustration of the device stack including the bulk heterojunction active layer (P3HT:PCBM or FTAZ:IT-M) and the electron transport layer that was either ZnO or PEIE. (b) Current-voltage relationship for P3HT:PCBM and FTAZ:IT-M OPV devices with a PEDOT:PSS or opaque metal electrode. (c) Transmittance of semitransparent electrodes ITO and PEDOT:PSS (FS30-1\%) and the overall transmittance of the semitransparent OPV devices with P3HT:PCBM and FTAZ:IT-M active layers. Inset: photograph of large-area transfer-printed FS30-1\% film on glass.

Table 2. OPV Device Characteristics Comparing Opaque Metal Electrodes to Semitransparent PEDOT:PSS (FS30-1\%) Electrodes for a Minimum of Four Devices

\begin{tabular}{|c|c|c|c|c|c|c|}
\hline active layer & top electrode & $V_{\mathrm{oc}}(\mathrm{V})$ & $J_{\mathrm{SC}}\left(\mathrm{mA} / \mathrm{cm}^{2}\right)$ & FF (\%) & ave PCE $(\%)^{a}$ & best PCE (\%) \\
\hline P3НT:РCBM & $\mathrm{MoO}_{3} / \mathrm{Ag}$ & $0.62 \pm 0.01$ & $8.74 \pm 0.4$ & $0.59 \pm 0.1$ & $3.18 \pm 0.2$ & 3.38 \\
\hline P3HT:PCBM & FS30-1\% & $0.62 \pm 0.01$ & $6.68 \pm 0.8$ & $0.50 \pm 0.1$ & $2.10 \pm 0.3$ & 2.38 \\
\hline FTAZ:IT-M & $\mathrm{MoO}_{3} / \mathrm{Al}$ & $0.96 \pm 0.002$ & $17.94 \pm 0.21$ & $0.66 \pm 0.1$ & $11.34 \pm 0.13$ & 11.60 \\
\hline FTAZ:IT-M & FS30-1\% & $0.91 \pm 0.03$ & $12.00 \pm 0.7$ & $0.51 \pm 0.003$ & $5.54 \pm 0.54$ & 6.02 \\
\hline
\end{tabular}

\section{CONCLUSIONS}

In this report, we demonstrate a transfer printing technique to print PEDOT:PSS top electrodes using an unpatterned elastomer stamp, named SHARP. The SHARP process consists of using a prestrained elastomer stamp. During the printing process, when the ink supported by the stamp is in contact with the receiving substrate, the prestrain in the stamp is reduced, resulting in a shear force at the stamp-ink interface. This shear force reduces the nominal work of adhesion between the stamp and ink, enabling the printing of polymer thin films. Various PEDOT:PSS formulations were used to explore the adhesion fracture energy limits of the transfer printing process. Using this material system, it was shown that SHARP is able to overcome a 10 times unfavorable adhesive fracture energy difference between the stamp/ink and ink/ receiver. The SHARP process was then used in the fabrication of high-performance semitransparent OPV cells. It was found that the transfer-printed PEDOT:PSS electrode significantly outperforms a film directly cast onto the device stack. This method is a generalized technique that can be used to print a wide range of polymer thin films. It is particularly useful in organic electronics where film morphology and interface structure are critical to device performance.

\section{ASSOCIATED CONTENT}

\section{Supporting Information}

The Supporting Information is available free of charge on the ACS Publications website at DOI: 10.1021/acsami.8b09968.

AFM images of a spun-cast and transfer-printed FS30$1 \%$ film and current-voltage characteristics of solutioncast semitransparent OPV cells (PDF)

SHARP technique (ZIP)

\section{AUTHOR INFORMATION}

\section{Corresponding Author}

*E-mail: btoconno@ncsu.edu.

ORCID $\odot$

Qianqian Zhang: 0000-0002-8896-1708

Wei You: 0000-0003-0354-1948

Harald Ade: 0000-0002-7871-1158

Brendan T. O’Connor: 0000-0002-8999-5184

Notes

The authors declare no competing financial interest.

\section{ACKNOWLEDGMENTS}

This work was supported by the National Science Foundation Award No. 1407885 and Award No. 1639429. The authors would like to thank Prof Yong Zhu's and Zheng Cui for 
assistance with contact angle measurements, and Prof Michael Dickey for assistance with the adhesion measurements.

\section{REFERENCES}

(1) Hur, S.-H.; Khang, D.-Y.; Kocabas, C.; Rogers, J. A. Nanotransfer Printing by Use of Noncovalent Surface Forces: Applications to ThinFilm Transistors That Use Single-Walled Carbon Nanotube Networks and Semiconducting Polymers. Appl. Phys. Lett. 2004, 85, 5730-5732.

(2) Liang, X.; Fu, Z.; Chou, S. Y. Graphene Transistors Fabricated via Transfer-Printing in Device Active-Areas on Large Wafer. Nano Lett. 2007, 7, 3840-3844.

(3) Loo, Y.-L.; Lang, D. V.; Rogers, J. A.; Hsu, J. W. P. Electrical Contacts to Molecular Layers by Nanotransfer Printing. Nano Lett. 2003, 3, 913-917.

(4) Loo, Y.-L.; Hsu, J. W. P.; Willett, R. L.; Baldwin, K. W.; West, K. W.; Rogers, J. A. High-Resolution Transfer Printing on GaAs Surfaces Using Alkane Dithiol Monolayers. J. Vac. Sci. Technol., B: Microelectron. Nanometer Struct.-Process., Meas., Phenom. 2002, 20, 2853.

(5) Kim, C.; Burrows, P. E.; Forrest, S. R. Micropatterning of Organic Electronic Devices by Cold-Welding. Science 2000, 288, 831-833.

(6) Forrest, S. R. The Path to Ubiquitous and Low-Cost Organic Electronic Appliances on Plastic. Nature 2004, 428, 911-918.

(7) Loo, Y.-L.; Willett, R. L.; Baldwin, K. W.; Rogers, J. A. Additive, Nanoscale Patterning of Metal Films with a Stamp and a Surface Chemistry Mediated Transfer Process: Applications in Plastic Electronics. Appl. Phys. Lett. 2002, 81, 562-564.

(8) Loo, Y.-L.; Willett, R. L.; Baldwin, K. W.; Rogers, J. A. Interfacial Chemistries for Nanoscale Transfer Printing. J. Am. Chem. Soc. 2002, 124, 7654-7655.

(9) Dai, A.; Zhou, Y.; Shu, A. L.; Mohapatra, S. K.; Wang, H.; Fuentes-Hernandez, C.; Zhang, Y.; Barlow, S.; Loo, Y.-L.; Marder, S. R.; Kippelen, B.; Kahn, A. Enhanced Charge-Carrier Injection and Collection via Lamination of Doped Polymer Layers p-Doped with a Solution-Processible Molybdenum Complex. Adv. Funct. Mater. 2014, 24, 2197-2204.

(10) Chabinyc, M. L.; Salleo, A.; Wu, Y.; Liu, P.; Ong, B. S.; Heeney, M.; McCulloch, I. Lamination Method for the Study of Interfaces in Polymeric Thin Film Transistors. J. Am. Chem. Soc. 2004, 126, 13928-13929.

(11) Carlson, A.; Bowen, A. M.; Huang, Y.; Nuzzo, R. G.; Rogers, J. A. Transfer Printing Techniques for Materials Assembly and Micro/ Nanodevice Fabrication. Adv. Mater. 2012, 24, 5284-5318.

(12) Yim, K.-H.; Zheng, Z.; Liang, Z.; Friend, R. H.; Huck, W. T. S.; Kim, J.-S. Efficient Conjugated-Polymer Optoelectronic Devices Fabricated by Thin-Film Transfer-Printing Technique. Adv. Funct. Mater. 2008, 18, 1012-1019.

(13) Tada, A.; Geng, Y.; Wei, Q.; Hashimoto, K.; Tajima, K. Tailoring Organic Heterojunction Interfaces in Bilayer Polymer Photovoltaic Devices. Nat. Mater. 2011, 10, 450-455.

(14) Wei, Q.; Tajima, K.; Hashimoto, K. Bilayer Ambipolar Organic Thin-Film Transistors and Inverters Prepared by the Contact-FilmTransfer Method. ACS Appl. Mater. Interfaces 2009, 1, 1865-1868.

(15) Chen, L.; Degenaar, P.; Bradley, D. D. C. Polymer Transfer Printing: Application to Layer Coating, Pattern Definition, and Diode Dark Current Blocking. Adv. Mater. 2008, 20, 1679-1683.

(16) Li, X.; Tang, X.; Ye, T.; Wu, D.; Wang, H.; Wang, X. Fully Printable Organic and Perovskite Solar Cells with Transfer-Printed Flexible Electrodes. ACS Appl. Mater. Interfaces 2017, 9, 1873018738.

(17) Brenckle, M. A.; Kaplan, D. L.; Omenetto, F. G. Direct Transfer Printing of Water Hydrolyzable Metals onto Silk Fibroin Substrates through Thermal-Reflow-Based Adhesion. Adv. Mater. Interfaces 2016, 3, 1600094 .

(18) Li, D.; Guo, L. J. Organic thin film transistors and polymer light-emitting diodes patterned by polymer inking and stamping. $J$. Phys. D: Appl. Phys. 2008, 41, 105115.

(19) Langowski, B. A.; Uhrich, K. E. Oxygen Plasma-Treatment Effects on Si Transfer. Langmuir 2005, 21, 6366-6372.
(20) Zhou, Y.; Khan, T. M.; Shim, J. W.; Dindar, A.; FuentesHernandez, C.; Kippelen, B. All-Plastic Solar Cells with a High Photovoltaic Dynamic Range. J. Mater. Chem. A 2014, 2, 3492-3497.

(21) Carlson, A.; Kim-Lee, H. J.; Wu, J.; Elvikis, P.; Cheng, H.; Kovalsky, A.; Elgan, S.; Yu, Q.; Ferreira, P. M.; Huang, Y.; Turner, K. T.; Rogers, J. A. Shear-Enhanced Adhesiveless Transfer Printing for Use in Deterministic Materials Assembly. Appl. Phys. Lett. 2011, 98, 264104.

(22) Kim-Lee, H.-J.; Carlson, A.; Grierson, D. S.; Rogers, J. A.; Turner, K. T. Interface Mechanics of Adhesiveless Microtransfer Printing Processes. J. Appl. Phys. 2014, 115, 143513.

(23) Cheng, H.; Wu, J.; Yu, Q.; Kim-Lee, H.-J.; Carlson, A.; Turner, K. T.; Hwang, K.-C.; Huang, Y.; Rogers, J. A. An Analytical Model for Shear-Enhanced Adhesiveless Transfer Printing. Mech. Res. Commun. 2012, 43, 46-49.

(24) O'Connor, B.; Kline, R. J.; Conrad, B. R.; Richter, L. J.; Gundlach, D.; Toney, M. F.; DeLongchamp, D. M. Anisotropic Structure and Charge Transport in Highly Strain-Aligned Regioregular Poly(3-Hexylthiophene). Adv. Funct. Mater. 2011, 21, 36973705.

(25) Gargi, D.; Kline, R. J.; DeLongchamp, D. M.; Fischer, D. A.; Toney, M. F.; O'Connor, B. T. Charge Transport in Highly Face-On Poly(3-hexylthiophene) Films. J. Phys. Chem. C 2013, 117, 1742117428.

(26) Awartani, O.; Kudenov, M. W.; O’Connor, B. T. Organic Photovoltaic Cells with Controlled Polarization Sensitivity. Appl. Phys. Lett. 2014, 104, 093306.

(27) Kitchen, B.; Awartani, O.; Kline, R. J.; McAfee, T.; Ade, H.; O'Connor, B. T. Tuning Open-Circuit Voltage in Organic Solar Cells with Molecular Orientation. ACS Appl. Mater. Interfaces 2015, 7, 13208-13216.

(28) Sun, T.; Scott, J. I.; Wang, M.; Kline, R. J.; Bazan, G. C.; O'Connor, B. T. Plastic Deformation of Polymer Blends as a Means to Achieve Stretchable Organic Transistors. Adv. Electron. Mater. 2017, 3,1600388 .

(29) Ro, H. W.; Akgun, B.; O'Connor, B. T.; Hammond, M.; Kline, R. J.; Snyder, C. R.; Satija, S. K.; Ayzner, A. L.; Toney, M. F.; Soles, C. L.; DeLongchamp, D. M. Poly(3-Hexylthiophene) and [6,6]-PhenylC61-Butyric Acid Methyl Ester Mixing in Organic Solar Cells. Macromolecules 2012, 45, 6587-6599.

(30) Herzing, A. A.; Ro, H. W.; Soles, C. L.; DeLongchamp, D. M. Visualization of Phase Evolution in Model Organic Photovoltaic Structures via Energy-Filtered Transmission Electron Microscopy. ACS Nano 2013, 7, 7937-7944.

(31) Awartani, O. M.; Zhao, B.; Currie, T.; Kline, R. J.; Zikry, M. A.; O'Connor, B. T. Anisotropic Elastic Modulus of Oriented Regioregular Poly(3-Hexylthiophene) Films. Macromolecules 2016, 49, 327-333.

(32) Gupta, D.; Wienk, M. M.; Janssen, R. A. J. Efficient Polymer Solar Cells on Opaque Substrates with a Laminated PEDOT:PSS Top Electrode. Adv. Energy Mater. 2013, 3, 782-787.

(33) Zhang, C.; Zhao, D.; Gu, D.; Kim, H.; Ling, T.; Wu, Y.-K. R.; Guo, L. J. An Ultrathin, Smooth, and Low-Loss Al-Doped Ag Film and Its Application as a Transparent Electrode in Organic Photovoltaics. Adv. Mater. 2014, 26, 5696-5701.

(34) Barr, M. C.; Howden, R. M.; Lunt, R. R.; Bulović, V.; Gleason, K. K. Top-illuminated Organic Photovoltaics on a Variety of Opaque Substrates with Vapor-printed Poly(3,4-ethylenedioxythiophene) Top Electrodes and MoO3Buffer Layer. Adv. Energy Mater. 2012, 2, 1404-1409.

(35) Lin, C.-F.; Liu, S.-W.; Hsu, W.-F.; Zhang, M.; Chiu, T.-L.; Wu, Y.; Lee, J.-H. Modification of silver anode and cathode for a topilluminated organic photovoltaic device. J. Phys. D: Appl. Phys. 2010, 43, 395101 .

(36) O’Connor, B.; An, K. H.; Pipe, K. P.; Zhao, Y.; Shtein, M. Enhanced Optical Field Intensity Distribution in Organic Photovoltaic Devices Using External Coatings. Appl. Phys. Lett. 2006, 89, 233502. 
(37) Patil, B. R.; Shanmugam, S.; Teunissen, J.-P.; Galagan, Y. AllSolution Processed Organic Solar Cells with Top Illumination. Org. Electron. 2015, 21, 40-46.

(38) Savva, A.; Neophytou, M.; Koutsides, C.; Kalli, K.; Choulis, S. A. Synergistic Effects of Buffer Layer Processing Additives for Enhanced Hole Carrier Selectivity in Inverted Organic Photovoltaics. Org. Electron. 2013, 14, 3123-3130.

(39) Zhou, Y.; Cheun, H.; Choi, S.; Fuentes-Hernandez, C.; Kippelen, B. Optimization of a Polymer Top Electrode for Inverted Semitransparent Organic Solar Cells. Org. Electron. 2011, 12, 827831.

(40) Fan, X.; Xu, B.; Liu, S.; Cui, C.; Wang, J.; Yan, F. TransferPrinted PEDOT:PSS Electrodes Using Mild Acids for High Conductivity and Improved Stability with Application to Flexible Organic Solar Cells. ACS Appl. Mater. Interfaces 2016, 8, 1402914036.

(41) Ouyang, J.; Chu, C.-W.; Chen, F.-C.; Xu, Q.; Yang, Y. HighConductivity Poly(3,4-Ethylenedioxythiophene):Poly(Styrene Sulfonate) Film and Its Application in Polymer Optoelectronic Devices. Adv. Funct. Mater. 2005, 15, 203-208.

(42) Baierl, D.; Fabel, B.; Lugli, P.; Scarpa, G. Efficient Indium-TinOxide (ITO) Free Top-Absorbing Organic Photodetector with Highly Transparent Polymer Top Electrode. Org. Electron. 2011, 12, $1669-1673$

(43) Savagatrup, S.; Chan, E.; Renteria-Garcia, S. M.; Printz, A. D.; Zaretski, A. V.; O'Connor, T. F.; Rodriquez, D.; Valle, E.; Lipomi, D. J. Plasticization of PEDOT:Plasticization of PEDOT:PSS by Common Additives for Mechanically Robust Organic Solar Cells and Wearable Sensors. Adv. Funct. Mater. 2015, 25, 427-436.

(44) Po, R.; Carbonera, C.; Bernardi, A.; Tinti, F.; Camaioni, N. Polymer- and Carbon-Based Electrodes for Polymer Solar Cells: Toward Low-Cost, Continuous Fabrication over Large Area. Sol. Energy Mater. Sol. Cells 2012, 100, 97-114.

(45) Okuzaki, H.; Harashina, Y.; Yan, H. Highly Conductive PEDOT/PSS Microfibers Fabricated by Wet-Spinning and DipTreatment in Ethylene Glycol. Eur. Polym. J. 2009, 45, 256-261.

(46) Takano, T.; Masunaga, H.; Fujiwara, A.; Okuzaki, H.; Sasaki, T. PEDOT Nanocrystal in Highly Conductive PEDOT:PSS Polymer Films. Macromolecules 2012, 45, 3859-3865.

(47) Wilson, P.; Lekakou, C.; Watts, J. F. In-Plane Conduction Characterisation and Charge Transport Model of DMSO Co-Doped, Inkjet Printed Poly(3,4-Ethylenedioxythiophene): Polystyrene Sulfonate (PEDOT:PSS). Org. Electron. 2013, 14, 3277-3285.

(48) Chen, J.-G.; Wei, H.-Y.; Ho, K.-C. Using Modified Poly(3,4Ethylene Dioxythiophene): Poly(Styrene Sulfonate) Film as a Counter Electrode in Dye-Sensitized Solar Cells. Sol. Energy Mater. Sol. Cells 2007, 91, 1472-1477.

(49) Yin, L.; Zhao, Z.; Jiang, F.; Li, Z.; Xiong, S.; Zhou, Y. PEDOT:PSS Top Electrode Prepared by Transfer Lamination Using Plastic Wrap as the Transfer Medium for Organic Solar Cells. Org. Electron. 2014, 15, 2593-2598.

(50) Kim, N.; Kang, H.; Lee, J.-H.; Kee, S.; Lee, S. H.; Lee, K. Highly Conductive All-Plastic Electrodes Fabricated Using a Novel Chemically Controlled Transfer-Printing Method. Adv. Mater. 2015, 27, 2317-2323.

(51) Jiang, F.; Liu, T.; Zeng, S.; Zhao, Q.; Min, X.; Li, Z.; Tong, J.; Meng, W.; Xiong, S.; Zhou, Y. Metal electrode-free perovskite solar cells with transfer-laminated conducting polymer electrode. Opt. Express 2015, 23, A83-A91.

(52) Xiong, S.; Tong, J.; Mao, L.; Li, Z.; Qin, F.; Jiang, F.; Meng, W.; Liu, T.; Li, W.; Zhou, Y. Double-Side Responsive Polymer nearInfrared Photodetectors with Transfer-Printed Electrode. J. Mater. Chem. C 2016, 4, 1414-1419.

(53) Hau, S. K.; Yip, H.-L.; Zou, J.; Jen, A. K.-Y. Indium Tin OxideFree Semi-Transparent Inverted Polymer Solar Cells Using Conducting Polymer as Both Bottom and Top Electrodes. Org. Electron. 2009, 10, 1401-1407.

(54) O'Connor, T. F.; Zaretski, A. V.; Shiravi, B. A.; Savagatrup, S.; Printz, A. D.; Diaz, M. I.; Lipomi, D. J. Stretching and Conformal
Bonding of Organic Solar Cells to Hemispherical Surfaces. Energy Environ. Sci. 2014, 7, 370-378.

(55) Kim, J. K.; Veerappan, G.; Heo, N.; Wang, D. H.; Park, J. H. Efficient Hole Extraction from Sb2S3 Heterojunction Solar Cells by the Solid Transfer of Preformed PEDOT:PSS Film. J. Phys. Chem. C 2014, 118, 22672-22677.

(56) Meitl, M. A.; Zhu, Z.-T.; Kumar, V.; Lee, K. J.; Feng, X.; Huang, Y. Y.; Adesida, I.; Nuzzo, R. G.; Rogers, J. A. Transfer Printing by Kinetic Control of Adhesion to an Elastomeric Stamp. Nat. Mater. 2006, 5, 33-38.

(57) Zhou, Y.; Cheun, H.; Choi, S.; Potscavage, W. J.; FuentesHernandez, C.; Kippelen, B. Indium Tin Oxide-Free and Metal-Free Semitransparent Organic Solar Cells. Appl. Phys. Lett. 2010, 97, 153304.

(58) Li, Z.; Meng, W.; Tong, J.; Zhao, C.; Qin, F.; Jiang, F.; Xiong, S.; Zeng, S.; Xu, L.; Hu, B.; Zhou, Y. A Nonionic Surfactant Simultaneously Enhancing Wetting Property and Electrical Conductivity of PEDOT:PSS for Vacuum-Free Organic Solar Cells. Sol. Energy Mater. Sol. Cells 2015, 137, 311-318.

(59) Lipomi, D. J.; Lee, J. A.; Vosgueritchian, M.; Tee, B. C.-K.; Bolander, J. A.; Bao, Z. Electronic Properties of Transparent Conductive Films of PEDOT:PSS on Stretchable Substrates. Chem. Mater. 2011, 24, 373-382.

(60) Chen, C.-h.; LaRue, J. C.; Nelson, R. D.; Kulinsky, L.; Madou, M. J. Electrical conductivity of polymer blends of poly $(3,4-$ ethylenedioxythiophene): Poly(styrenesulfonate): N-methyl-2-pyrrolidinone and polyvinyl alcohol. J. Appl. Polym. Sci. 2012, 125, 31343141.

(61) Kim, J. Y.; Jung, J. H.; Lee, D. E.; Joo, J. Enhancement of Electrical Conductivity of Poly(3,4-Ethylenedioxythiophene)/Poly(4Styrenesulfonate) by a Change of Solvents. Synth. Met. 2002, 126, $311-316$.

(62) Kendall, K. Thin-Film Peeling-the Elastic Term. J. Phys. D: Appl. Phys. 1975, 8, 1449-1452.

(63) Zhou, Y.; Fuentes-Hernandez, C.; Shim, J.; Meyer, J.; Giordano, A. J.; Li, H.; Winget, P.; Papadopoulos, T.; Cheun, H.; Kim, J.; Fenoll, M.; Dindar, A.; Haske, W.; Najafabadi, E.; Khan, T. M.; Sojoudi, H.; Barlow, S.; Graham, S.; Bredas, J.-L.; Marder, S. R.; Kahn, A.; Kippelen, B. A Universal Method to Produce Low-Work Function Electrodes for Organic Electronics. Science 2012, 336, 327332.

(64) Sun, Y.; Seo, J. H.; Takacs, C. J.; Seifter, J.; Heeger, A. J. Inverted Polymer Solar Cells Integrated with a Low-TemperatureAnnealed Sol-Gel-Derived ZnO Film as an Electron Transport Layer. Adv. Mater. 2011, 23, 1679-1683.

(65) Price, S. C.; Stuart, A. C.; Yang, L.; Zhou, H.; You, W. Fluorine Substituted Conjugated Polymer of Medium Band Gap Yields 7\% Efficiency in Polymer-Fullerene Solar Cells. J. Am. Chem. Soc. 2011, $133,4625-4631$.

(66) Lee, I.; Kim, G. W.; Yang, M.; Kim, T.-S. Simultaneously Enhancing the Cohesion and Electrical Conductivity of PEDOT:PSS Conductive Polymer Films Using DMSO Additives. ACS Appl. Mater. Interfaces 2016, 8, 302-310. 